\title{
The Influence of Tail Autotomy on the Escape Response of the Cape Dwarf Gecko, Lygodactylus capensis
}

\author{
Katarina Medger*`, Luke Verburgt* \& Philip W. Bateman* \\ * Department of Zoology and Entomology, University of Pretoria, Pretoria, South \\ Africa \\ $\dagger$ Institute of Zoology, Martin-Luther-University Halle-Wittenberg, Germany \\ Correspondence to P. W. Bateman, Department of Zoology \& Entomology, \\ University of Pretoria, Pretoria 0002, South Africa. \\ E-mail: pwbateman@zoology.up.ac.za
}

\section{Abstract}

Tail autotomy as a defence against predators occurs in many species of lizard. Although tail autotomy may provide an immediate benefit in terms of survival it may nevertheless be costly due to other functions of the tail. For example, tail autotomy may affect the locomotory performance of lizards during escape. We investigated the influence of tail autotomy on the escape performance of the Cape Dwarf Gecko, Lygodactylus capensis, on a vertical and a horizontal surface. Autotomized geckos were significantly slower than intact geckos during vertical escape, whereas tail autotomy did not influence the horizontal escape speed. Backward falling of the autotomized geckos on the vertical platform may explain the reduced speed. In addition, tail autotomy did not significantly affect body curvature and stride length of the geckos. The observed decrease of escape speed on a vertical platform may influence the habitat use and behaviour of these geckos. Ecological consequences resulting from tail autotomy are discussed in light of these findings.

\section{Introduction}

Many animals sacrifice body parts in response to predation or in antagonistic encounters with conspecifics. This phenomenon, known as autotomy, can enable the individual to escape such encounters (Vitt et al. 1977; Formanowicz 1990; Ducey et al. 1993). Autotomy occurs across many taxa and many different body parts can be shed (reviewed in Maginnis 2006). Lamellae autotomy, for example, can be found in damselfly larvae (Stoks 1998) and limb autotomy in starfish (Ramsay et al. 2001), crustaceans (Juanes \& Smith 1995; Wasson et al. 2002), insects (Bateman \& Fleming 2005) and arachnids (Formanowicz 1990; Brueseke et al. 2001). Some vertebrates are able to shed the whole tail or parts of it, fracturing the tail at distinct regions of weakness (Arnold 1984). Caudal (tail) autotomy can be found in a few rodents (Dubost \& Gasc 1987; McKee \& Adler 2002), snakes (Arnold 1984), some salamanders (Maiorana 1977; Ducey et al. 1993) and most commonly in numerous lizard species (Vitt et al. 1977; Arnold 1984). 
Caudal autotomy is an extreme escape strategy which can benefit the lizard in two ways: it enables escape from the predator and may divert the predator's attention from the lizard's body (Arnold 1988). For example, this strategy increases the chance of surviving a potentially fatal attack for tailed geckos, Coleonyx variegatus, which were more likely to survive an encounter with snakes, Hypsiglena ochrorhyncha, than tailless individuals (Congdon et al. 1974).

Although tail autotomy may provide an immediate benefit in terms of escaping a predator, it has nevertheless been shown to be costly in multiple families of lizards because of the numerous functions of the tail, e.g. use as a weapon, for social behaviour, storage of energy reserves and locomotion (balance, climbing and swimming; Arnold 1984). Autotomy costs may vary between species, ontogenetic stages, and habitat use and because of short-term changes within an individual depending on the function of the tail (Arnold 1988). Tail loss may result, for example, in reduced social status and mating success (Martín \& Salvador 1993; Salvador et al. 1995), which in turn may lead to lower reproductive success and lower survival (Fox \& Rostker 1982; Fox \& McCoy 2000), resulting in reduced lifetime fitness. Other costs incurred may be loss of energy reserves stored in the tail and energy needed for tail regeneration (Vitt \& Cooper 1986; McConnachie \& Whiting 2003). Tail autotomy may also result in a higher vulnerability to further predation until the tail is regenerated (Congdon et al. 1974; Downes \& Shine 2001). Lizards may compensate for costs resulting from autotomy, with a change of behaviour and habitat use. Tailless individuals, for example, reduce home range size (Salvador et al. 1995), flee earlier (Cooper 2003), increase use of safer microhabitats (Martín \& Salvador 1992; Cooper 2003) and carry out cryptic anti-predator behaviour (Formanowicz et al. 1990).

Lizard locomotory ability may be influenced by tail autotomy such that it incurs a major cost for the individual by reducing the chance of escaping from a predator (Arnold 1984). Nevertheless, locomotory costs may be very different for different species or even be non-existent if the tail is not an important element used for locomotion. Tail loss decreased, for example, the swimming abilities of the water skink Sphenomorphus quoyii but in contrast its running speed was only slightly affected (Daniels 1985). Some studies found an increase in sprint speed with tail loss (Daniels 1983) while others found a decrease (Formanowicz et al. 1990; Chapple et al. 2004) or no change in speed with tail loss (McConnachie \& Whiting 2003). A decrease in speed during spontaneous movement, slower escape speed and shorter escape distance, and a reduction in stride length after tail loss were demonstrated for Psammodromus algirus, a ground-dwelling lizard (Martín \& Avery 1998).

Most studies on tail autotomy and its influence on the locomotion of lizards have concentrated on ground-dwelling lizards. However, Brown et al. (1995) examined the running speed and the arboreal locomotion of the wall lizard Podarcis muralis with tail loss and found an increase in running speed and distance, but a decrease in speed and distance during arboreal locomotion with the loss of the tail. These results indicate that the tail of this species plays different roles in horizontal locomotion and arboreal locomotion. Nevertheless, the specific role of the tail during climbing is not actually known, particularly for geckos which are able to climb on smooth surfaces. This study aims to compare the escape performance of the dwarf gecko Lygodactylus capensis on vertical and horizontal surfaces before and after tail autotomy. 
Furthermore, we aim to determine the importance of the tail for climbing on vertical surfaces and the locomotion costs which arise from tail loss for this gecko.

Lygodactylus capensis is a small, diurnal gecko occurring in southern and eastern Africa. It is characterized by a disc-like enlargement at the end of the feet with adhesive hairs or setae which permit the climbing of smooth vertical surfaces because of a very high adhesive force originating from van der Waals forces (Autumn et al. 2000). Moreover, these geckos also have an adhesive pad at the tail tip which is similar to the adhesive structures at the feet (Bauer 1998) and is used as a fifth point for attachment (Vitt \& Ballinger 1982). The adhesive hairs at the feet and the adhesive tail tip are an adaptation to the arboreal habits of this gecko which can often be found in trees high above ground (Pianka \& Huey 1978). Furthermore, this species also occurs on house walls, fences and lamp posts in urban areas. The results for horizontal and vertical locomotion and the influence of tail autotomy will be used to predict ecological and behavioural consequences for this climbing gecko resulting from tail loss.

We predicted a decrease in escape speed when the geckos autotomize the tail. Furthermore, we predicted that the geckos would be slower on a vertical structure than on a horizontal one, because vertical locomotion is influenced more by gravity than horizontal locomotion. As the tail is often used to counterbalance the body in lizards and reduces the lateral swing of the hindquarters (Arnold 1984), it was predicted that the curvature of the body should be less in geckos with intact tails than in geckos with autotomized tails. Furthermore, if the geckos increased speed by increasing stride length (as shown for P. algirus; Martín \& Avery 1998), stride length would be greater during horizontal escape than during vertical escape and it would also be greater for intact than for autotomized geckos.

\section{Materials and Methods}

\section{Experimental Animals}

Lygodactylus capensis were caught by hand in Pretoria in suburban gardens and on the University of Pretoria campus. Individual geckos were kept separately in 21 clear plastic jars with a natural photoperiod. In addition, a lamp provided low UV light. The temperature in the room was relatively stable $\left(20-25^{\circ} \mathrm{C}\right)$ and did not require an additional heat source. Geckos were fed daily with cricket nymphs, ants or termites dusted with multivitamin powder, and water was provided daily.

Sex, intact and autotomized body mass (g), snout-vent length (SVL; mm), total body length $(\mathrm{mm})$, tail length $(\mathrm{mm})$, mass $(\mathrm{g})$ and length $(\mathrm{mm})$ of the autotomized tail and the stump length $(\mathrm{mm})$ were recorded for each gecko. Furthermore, it was recorded whether the geckos possessed an original or regenerated tail prior to experimental autotomy.

\section{Experimental Equipment and Measurements}

The escape performance on both vertical and horizontal platforms was measured for L. capensis when intact and when autotomized. Twenty-one geckos (13 females and eight males) were used for the escape performance on the vertical platform and 23 different geckos (nine females and 14 males) were used for the escape performance on the horizontal platform. However, not all animals yielded useable data for all 
escape performance variables. Pregnant females were excluded as pregnancy may influence escape performance (Chapple \& Swain 2002).

The running platform, on which geckos were tested individually, consisted of a smooth plastic board $(54 \times 39 \mathrm{~cm})$ marked with a grid of half centimetre divisions and was used for both vertical and horizontal escape performance. For vertical escape, the platform was slightly inclined at a mean angle of $2.8 \pm 0.4^{\circ}$ from vertical and was suspended over a 1001 plastic tub which prevented escape if the gecko fell or jumped. For horizontal escape, the platform was laid horizontally over two tubs. The temperature in this room was held constant at $27 \pm 0.3^{\circ} \mathrm{C}$. The geckos were housed in the experimental room for $1 \mathrm{~h}$ before the onset of experimental trials, allowing for acclimatization to experimental conditions. Geckos were placed on the running platform by hand and covered with a semi-circular plastic covering which ensured a straight line of flight. Escape was induced by tapping the base of the tail, following Martín \& Avery (1998). The entire procedure after placing the gecko on the platform and covering it to inducing flight lasted $<2 \mathrm{~s}$.

The escape performance of the geckos was recorded on video with a Sony DCR-TRV $22 \mathrm{E}$ video-camera (25 frames/s) (Sony, Tokyo, Japan) from which individual video frames were extracted to digital images. These frames were used to measure escape speeds $(\mathrm{cm} / \mathrm{s})$, body curvature $\left(^{\circ}\right)$ and stride length $\left(^{\circ}\right)$ of the individual geckos for both platform orientations. Escape distance $(\mathrm{cm})$ and escape time $(\mathrm{s})$ were measured to calculate the escape speeds. Escape distance was defined as the distance between the initial position of the snout and its position in the first pause after fleeing. Escape time was measured as the time interval between the initial position and the final position of the snout (resolution $=0.04 \mathrm{~s}$ ). Overall speed, initial speed and maximum speed were calculated from this data. Overall speed was determined over the whole escape distance. Initial speed was calculated over the first three frames $(0.08 \mathrm{~s})$ and maximum speed was recorded as the highest speed between two frames $(0.04 \mathrm{~s})$ during the entire escape.

The curvature of the body and the stride length were estimated frame-by-frame with custom-designed software (developed by L. Verburgt). Body curvature was measured as the angle between the perpendicular lines to the thoracic girdle axis and to the pelvic girdle axis (Fig. 1). The angle of the body was measured for four different frames (non-randomly selected for precise measurements) and the smallest angle of these was selected as the maximum body curvature for each individual gecko.

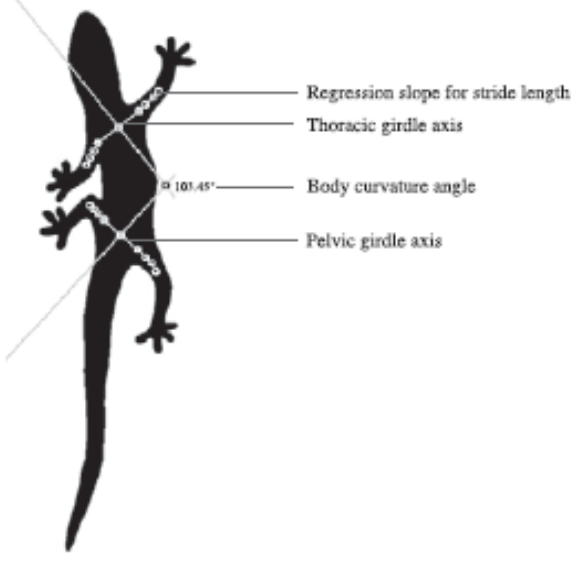


Fig. 1: Body curvature $\left(^{\circ}\right)$ and stride-length $\left(^{\circ}\right)$ measurements obtained from customdesigned software. Four positional markers (dots) were used to calculate the regression slope of each leg separately. Thoracic and pelvic girdle axes were then approximated by a straight line between the inner-most of the four dots per leg (grey dots). The body curvature angle was then calculated between the perpendicular lines to the thoracic and pelvic girdle.

To determine the stride length, the slope of each limb in two successive frames was measured and the angle between the successive limb positions was calculated (Fig. 1). This angle was used as a relative measure of stride length that accounts for differences in body size and limb length (Martín \& Avery 1998). Two stride-length measurements were performed for each separate limb on different frames. The mean values for the front limbs and the hind limbs were calculated from the angle of the appropriate right and left limbs and from these two stride-length measurements the maximum stride length was chosen. The stride length of front and hind limbs was considered separately.

Every body curvature and stride-length measurement was repeated and the mean of these repeated measurements was used as the dependent variable for statistical analyses to reduce measurement errors.

The frequency of backward falls was recorded for escape performance on the vertical platform. Backward falling was defined when a gecko's body fell back from the platform such that the front legs were fully extended during flight. Video frame sequences where the front legs were not touching the surface were not used because geckos usually fell from the platform.

\section{Experimental Structure}

Each escape performance experiment (vertical and horizontal) was performed with the intact individuals (control treatment) and the individuals with autotomized tails (autotomized treatment). Two trials were carried out for every treatment on different days, allowing for the quantification of within-individual variation.

Intact geckos were allowed to rest for $2 \mathrm{~d}$ and autotomized geckos for $3 \mathrm{~d}$ between the trials. The tails of the geckos were autotomized with forceps by grasping the gecko $5 \mathrm{~mm}$ from the base of the tail. Geckos were held like this until the tail was shed. Tail autotomy was induced $10 \mathrm{~d}$ after each individual completed both trials of the intact experiment. Geckos were allowed to recover for $2 \mathrm{~d}$ following tail autotomy before they were subjected to the escape performance experiments again. This time was chosen to let the geckos recover from autotomy stress ( $2 \mathrm{~d}$ after tail autotomy, plasma corticosterone levels in water skinks, Eulamprus heatwolei, have reduced to levels equivalent to those measured $14 \mathrm{~d}$ post-autotomy treatment or to levels measured in control animals, Langkilde \& Shine 2006), although it was not long enough to allow re-growth of the tail.

Speed variables, body curvature and stride length were measured for all trials of both the vertical and the horizontal escape performance experiment. However, overall speed was excluded from the measurements of the horizontal experiment because it 
could not be calculated reliably as geckos frequently ran the entire distance of the platform in a single escape burst and consequently, it was impossible to calculate escape distance and escape time. Speed variables were measured for straight flights (approximately perpendicular to platform $\mathrm{x}$-axis) on the vertical platform to prevent erroneous measurements because of easier running or different running mechanisms during a non-straight flight. In the case of multiple escape bursts, the maximum value of each speed variable was selected for each individual gecko for every trial of both escape performance experiments.

\section{Statistical Analysis}

Repeated measures anova (RM-anova) was used to compare the escape speed variables, body curvature and stride length (front and hind legs) between intact and autotomized treatments and between the vertical and the horizontal escape performance experiments (except overall speed). The within-effects were Treatment and Trial where Trial was nested within Treatment for all variables and experiment (vertical and horizontal) was the between subjects factor. The frequency of backward falls on the vertical platform was compared between intact and autotomized geckos as well (Yates-corrected chi-squared test).

Differences in escape performance variables between males and females and between geckos with original tails and with regenerated tails (between subjects factor) were analysed with a RM-anova. For these analyses, the maximum value of both trials was used to compare the most expressive escape performances. A t-test was applied to compare morphological characteristics between males and females as well as between original and regenerated tails.

The eight morphological characteristics measured were subjected to a principal components analysis (PCA). Following this, regression analyses were performed to establish linear relationships between the first principle component extracted from the PCA and the escape performance variables for intact individuals. Regression analyses were performed to determine linear relationships between the speed variables and body curvature or stride length for intact geckos and furthermore, to determine relationships between overall speed and maximum speed for both treatments. The mean value of the two trials was used for all regression analyses.

A sequential Bonferroni correction for multiple comparisons (SqBc; Rice 1989) was applied for each analysis performed. The Bonferroni level of significance is reported as italicized values in brackets adjacent to the lowest computed p-value, where applicable. Levene's test for homogeneity of variance was used to test for homoscedasticity. These statistical analyses were performed with statistica version 6.0 (StatSoft Inc. 2001). All data are given as the mean \pm 1 SD.

\section{Results}

\section{Morphology}

The morphological characteristics (total body length, SVL, intact and autotomized body mass, tail length and tail mass) did not differ between the sexes [all $\mathrm{t}_{42}<2.33$; $\mathrm{p}>0.02$; autotomized body mass: $\mathrm{p}=0.02(0.15)]$ after a SqBc. Original tails were significantly longer than regenerated tails $\left[\mathrm{t}_{42}=4.03 ; \mathrm{p}=0.0002(0.001)\right.$, original tail 
length $(n=20): 37.9 \pm 3.0 \mathrm{~mm}$; regenerated tail length $(\mathrm{n}=24): 32.5 \pm 5.2 \mathrm{~mm}]$. The other morphological characteristics (total body length, SVL, intact and autotomized body mass and tail mass) did not differ between geckos with original or regenerated tails after a SqBc [all $\left.t_{42}<2.60 ; p>0.01, \mathrm{SVL}: \mathrm{p}=0.013(0.06)\right]$. Differences in speed variables, body curvature and stride length (front and hind) between males and females and between geckos with original and regenerated tails are presented in Table 1.

Table 1: Differences in speed variables, body curvature and stride length between the sexes (males and females) and between tail types (original and regenerated) established by repeated measures anova

\begin{tabular}{|c|c|c|c|c|c|c|c|c|c|}
\hline \multirow[b]{3}{*}{ Dependent variable } & \multirow[b]{3}{*}{ df } & \multicolumn{4}{|c|}{$\operatorname{sex}$} & \multicolumn{4}{|c|}{ Tall type } \\
\hline & & \multicolumn{2}{|c|}{ n } & \multirow[b]{2}{*}{$F$} & \multirow[b]{2}{*}{ p-value } & \multicolumn{2}{|c|}{$\mathrm{n}$} & \multirow[b]{2}{*}{$\mathrm{F}$} & \multirow[b]{2}{*}{ p-value } \\
\hline & & Males & Females & & & Original & Regenerated & & \\
\hline Overall speed $\mathrm{km} / \mathrm{s}$ ) & 1,14 & 7 & 9 & 3.88 & 0.07 & 9 & 7 & 5.86 & $0.03 *$ \\
\hline Maximum speed $(\mathrm{cm} / \mathrm{s})$ & 1,35 & 21 & 18 & 0.14 & 0.71 & 19 & 20 & 0.37 & 0.55 \\
\hline Inital speed $(\mathrm{cm} / \mathrm{s})$ & 1,31 & 19 & 16 & 0.77 & 0.39 & 17 & 18 & 1.14 & 0.29 \\
\hline Body curvature 19 & 1,25 & 16 & 13 & 2.03 & 0.17 & 16 & 13 & 0.02 & 0.90 \\
\hline Stride length front legs 19 ) & 1,22 & 15 & 11 & 0.61 & 0.44 & 16 & 10 & 0.07 & 0.79 \\
\hline Stride length hind legs 19 ) & 1,25 & 16 & 13 & 4.07 & 0.05 & 16 & 13 & 1.29 & 0.27 \\
\hline
\end{tabular}

Statistical results are shown for the between-effect (sex or tail type) only. p-Values which were no longer significant after a sequential Bonferroni correction are indicated with an asterisk $(*)$.

Principal component analysis collapsed the eight morphological measurements into three principal components (Table 2). Only the first principal component (PC1) was used for further analyses as a single measure of size. PC1 did not explain variation in the speed variables, body curvature or stride length (front and hind legs) for both escape performance experiments (Table 3). Maximum speed was not related to overall speed for the intact geckos $\left(\mathrm{F}_{1,14}=3.41\right.$; adjusted $\left.\mathrm{R}^{2}=0.14 ; \mathrm{p}=0.09\right)$ but was for the autotomized geckos $\left(\mathrm{F}_{1,14}=59.6\right.$; adjusted $\left.\mathrm{R}^{2}=0.80 ; \mathrm{p}<0.01\right)$ for the vertical experiment. Maximum speed and overall speed were not related to body curvature or stride length for both the vertical and horizontal experiment (Table 4). Body curvature and stride length could not be measured for initial speed due to obstruction by the semi-circular plastic covering and therefore, we could not determine the relationships between these variables. As tail type, sex or morphological characteristics did not affect escape performance variables, pooling of the data for further analysis was justified. 
Table 2: Principle component analysis of the morphological measurements for Lygodactylus capensis

\begin{tabular}{|c|c|c|c|c|c|c|}
\hline \multirow[b]{3}{*}{ Variable } & \multicolumn{6}{|c|}{ Component } \\
\hline & \multicolumn{3}{|c|}{ Vertical } & \multicolumn{3}{|c|}{ Horizontal } \\
\hline & 1 & 2 & 3 & 1 & 2 & 3 \\
\hline Snout-vent length $(33.6 \pm 2.1 \mathrm{~mm}$, range: $29.0-38.2)$ & 0.66 & -0.60 & - & 0.58 & - & - \\
\hline Tall length $135.0 \pm 5.1 \mathrm{~mm}$, range: $22.9-42.7)$ & 0.72 & 0.69 & - & 0.89 & - & - \\
\hline Total body length $(60.6 \pm 5.8 \mathrm{~mm}$, range: $53.5-79.6)$ & 0.92 & - & - & 0.95 & - & - \\
\hline Autotomized tai length $(29.8 \pm 5.0 \mathrm{~mm}$, range: $18.1-37.9)$ & 0.71 & 0.67 & - & 0.89 & - & - \\
\hline Intact body mass $(1.1 \pm 0.2 \mathrm{~g}$, range: $0.7-1.5)$ & 0.85 & - & - & 0.67 & 0.69 & - \\
\hline Autotomized body mass $10.9 \pm 0.2 \mathrm{~g}$, range: $0.6-1.4)$ & 0.80 & -0.53 & - & 0.56 & 0.79 & - \\
\hline Tall mass $(0.1 \pm 0.03 \mathrm{~g}$, range: $0.08-0.2)$ & 0.93 & - & - & 0.77 & - & - \\
\hline Sump length $15.1 \pm 0.8 \mathrm{~mm}$, range: $3.6-6.9)$ & - & - & 0.92 & - & - & 0.97 \\
\hline Egenvalues & 4.6 & 2.0 & 1.0 & 4.2 & 1.9 & 1.0 \\
\hline Cumulative variance explained (z) & 57.2 & 82.4 & 94.9 & 522. & 75.4 & 88.2 \\
\hline
\end{tabular}

Values are factor loadings (Pearson's correlation coefficient between components and the original variable). Mean $\pm \mathrm{SD}$ and the range are presented for each variable.

Table 3: The relationships between escape performance variables measured and principal component 1 (single morphological measure)

\begin{tabular}{|c|c|c|c|c|c|c|c|}
\hline \multirow[b]{2}{*}{ Experiment } & \multirow[b]{2}{*}{ Dependent varable } & \multicolumn{6}{|c|}{ Principal component 1} \\
\hline & & df & $\mathrm{F}$ & $\mathrm{R}^{2}$ & B & $\mathrm{t}$ & p-vate \\
\hline \multirow[t]{6}{*}{ Vertical } & Overall speed (cm/s) & 1,14 & 1.20 & 0.01 & 0.61 & 1.09 & 0.29 \\
\hline & Maximum speed $1 \mathrm{~cm} / \mathrm{s}$ & 1,14 & 0.53 & 0 & 1.13 & 0.73 & 0.48 \\
\hline & Intial speed (cm/s) & 1,14 & 0.29 & 0 & -0.46 & -0.54 & 0.60 \\
\hline & Body curvature 19 & 1,12 & 1.95 & 0.07 & -1.40 & -1.40 & 0.19 \\
\hline & Stride length front $\operatorname{legs}\left(l^{2}\right)$ & 1,10 & 3.15 & 0.16 & 1.20 & 1.78 & 0.11 \\
\hline & Stride length hind legs (1) & 1,12 & 0.32 & 0 & -0.34 & -0.57 & 0.58 \\
\hline \multirow[t]{5}{*}{ Horizontal } & Maximum speed (cm/s) & 1,21 & 0.26 & 0 & 0.55 & 0.51 & 0.62 \\
\hline & Intial speed (cm/s) & 1,17 & 0.65 & 0 & 1.07 & 0.81 & 0.43 \\
\hline & Body curvature 19 & 1,13 & 0.79 & 0 & 0.70 & 0.99 & 0.39 \\
\hline & Strice length front $\operatorname{logs} l^{2}$ ) & 1,12 & 0.02 & 0 & 0.10 & 0.15 & 0.88 \\
\hline & Strice length hind legs $\left({ }^{3}\right)$ & 1,13 & 0.07 & 0 & -0.16 & -0.26 & 0.80 \\
\hline
\end{tabular}

Relationships were determined by regression analyses and are shown for the intact treatment only as the autotomized treatment showed similar results.

Table 4: The relationships between speed variables and body curvature or stride length for intact geckos as determined by regression analyses (autotomized geckos showed similar results)

\begin{tabular}{|c|c|c|c|c|c|c|c|c|c|c|c|c|c|c|c|c|c|c|}
\hline \multirow[b]{3}{*}{ Independent variable } & \multicolumn{12}{|c|}{ Vertical } & \multicolumn{6}{|c|}{ Horizontal } \\
\hline & \multicolumn{6}{|c|}{ Maximum speed (cm/s) } & \multicolumn{6}{|c|}{ Overall speed (cm/s) } & \multicolumn{6}{|c|}{ Maximum speed (cm/s) } \\
\hline & df & $\mathrm{F}$ & $\mathrm{R}^{2}$ & B & $\mathrm{t}$ & $p-V a l u e$ & df & $\mathrm{F}$ & $\mathbb{R}^{2}$ & B & $\mathrm{t}$ & p-Value & $d f$ & $\mathrm{~F}$ & $\mathrm{R}^{2}$ & B & $\mathrm{t}$ & p-value \\
\hline Body curvature $(p)$ & 1,12 & 0.001 & 0 & -0.01 & -0.17 & 0.97 & 1,12 & 0.63 & 0 & -0.12 & -0.79 & 0.44 & 1,13 & 0.13 & 0 & 0.12 & 0.36 & 0.73 \\
\hline Stride length front legs 17 ) & 1,10 & 0.03 & 0 & -0.08 & -0.17 & 0.86 & 1,10 & 0.0003 & 0 & -0.005 & -0.02 & 0.99 & 1,12 & 1.68 & 0.05 & 0.58 & 1.30 & 0.22 \\
\hline Stride length hind legs 17 & 1,12 & 0.03 & 0 & 0.09 & 0.18 & 0.86 & 1,12 & 1.20 & 0.02 & -0.28 & -0.10 & 0.29 & 1,13 & 0.81 & 0 & 0.41 & 0.90 & 0.39 \\
\hline
\end{tabular}




\section{Escape Performance}

The initial speed data was heteroscedastic $\left(\mathrm{F}_{7,132}=2.24 ; \mathrm{p}=0.03\right)$ but after squareroot transformation it was homoscedastic $\left(\mathrm{F}_{7,132}=1.41 ; \mathrm{p}=0.2\right)$. Table 5 shows comparisons between intact and autotomized treatments and between vertical and horizontal experiments for the escape speed variables, body curvature and front and hind stride length. Furthermore, interactions between experiment and treatment for the escape performance variables are displayed in Table 5. Maximum speed and body curvature for intact and autotomized individuals and for the vertical and the horizontal experiment are presented in Figs 2 and 3, respectively.

Table 5: Comparison between intact and autotomized treatments for both the vertical and horizontal escape performance experiments $\left(\mathrm{p}_{1}\right)$ as well as comparison between the vertical and horizontal escape performance experiments within a treatment $\left(\mathrm{p}_{2}\right)$ for speed variables, body curvature and stride length of Lygodactylus capensis

\begin{tabular}{|c|c|c|c|c|c|c|c|c|c|c|c|c|c|c|}
\hline \multirow[b]{2}{*}{ Dependent variable } & \multirow{2}{*}{\multicolumn{2}{|c|}{$n$ df }} & \multirow{2}{*}{\multicolumn{2}{|c|}{ Experiment Intact }} & \multirow[b]{2}{*}{ Autotomized } & \multicolumn{3}{|c|}{ Treatment (f vs. A) } & \multirow[b]{2}{*}{ Treatment } & \multicolumn{3}{|c|}{ Experment (V vs. H) } & \multicolumn{2}{|c|}{$\begin{array}{l}\text { Treat- } \\
\text { ment*Experi- } \\
\text { ment }\end{array}$} \\
\hline & & & & & & $\mathrm{F}$ & p-value & pr-value & & $\mathrm{F}$ & pralue & parvalue & $\mathrm{F}$ & prvalue \\
\hline $\begin{array}{l}\text { Overall speed } \\
(\mathrm{cm} / \mathrm{s})\end{array}$ & 16 & 1,15 & $v$ & $36.8 \pm 6.4$ & $29.6 \pm 73$ & 43.65 & $<0.001$ & - & - & - & - & - & - & - \\
\hline \multirow{2}{*}{$\begin{array}{l}\text { Maximum speed } \\
(\mathrm{cm} / \mathrm{s})\end{array}$} & 16 & 1,37 & $v$ & $59.5 \pm 9.9$ & $49.7 \pm 9.3$ & 7.92 & 0.02 & $<0.01$ & 1 & 119.5 & 4.001 & 4.001 & 7.92 & $<0.01$ \\
\hline & 23 & & $\mathrm{H}$ & $80.8 \pm 13.1$ & $81.6 \pm 14.5$ & & & 0.98 & A & & & 4.001 & & \\
\hline \multirow{2}{*}{ Inital speed $(\mathrm{cm} / \mathrm{s})$} & 16 & 1,33 & $v$ & $35.9 \pm 10.9$ & $23.9 \pm 93$ & 6.67 & 0.01 & $<0.01$ & 1 & 15.85 & $\$ .001$ & 0.88 & 12.65 & $<0.01$ \\
\hline & 19 & & H & $39.5 \pm 15.5$ & $41.8 \pm 15.1$ & & & 0.89 & A & & & 4.001 & & \\
\hline \multirow[t]{2}{*}{ Body curvature 19} & 14 & 1,27 & $v$ & $111.6 \pm 11.0$ & $1092 \pm 12.2$ & 2.06 & 0.32 & 0.85 & 1 & 35.92 & $\$ .001$ & 4.001 & 4.71 & 0.04 \\
\hline & 15 & & H & $94.0 \pm 9.9$ & $100.8 \pm 10.4$ & & & 0.12 & A & & & 0.05 & & \\
\hline \multirow{2}{*}{$\begin{array}{l}\text { Stride length } \\
\left.\text { front legs }\left.\right|^{2}\right\rangle\end{array}$} & 12 & 1,24 & $v$ & $78.4 \pm 6.3$ & $78.0 \pm 5.7$ & 0.14 & 0.16 & 1 & 1 & 0.02 & 0.89 & 0.87 & 1.25 & 0.27 \\
\hline & 14 & & H & $80.0 \pm 6.7$ & $76.9 \pm 7.7$ & & & 0.26 & A & & & 0.94 & & \\
\hline \multirow{2}{*}{$\begin{array}{l}\text { Stride length } \\
\text { hind legs }\left(p^{p}\right)\end{array}$} & 14 & 1,27 & v & $78.6 \pm 7.1$ & $77.3 \pm 10.4$ & 1.04 & 0.71 & 0.91 & 1 & 0.48 & 0.49 & 0.68 & 1.79 & 0.19 \\
\hline & 15 & & $\mathrm{H}$ & $755 \pm 72$ & $77.7 \pm 7.9$ & & & 0.61 & A & & & 1 & & \\
\hline
\end{tabular}

F- and p-values for comparisons between treatments, experiments and interaction of treatment and experiment are obtained from a repeated measures anova (RM-anova). $\mathrm{p}_{1}$ - and $\mathrm{p}_{2}$-values originate from a Tukey-test following a RM-anova except for overall speed where post hoc comparison was not required. The horizontal experiment is designated with ' $\mathrm{H}$ ' and the vertical experiment with ' $\mathrm{V}$ '. Intact treatment (control) is indicated with 'I' and autotomized treatment with 'A'. Mean values are presented $\pm 1 \mathrm{SD}$ and significant differences are indicated in bold.

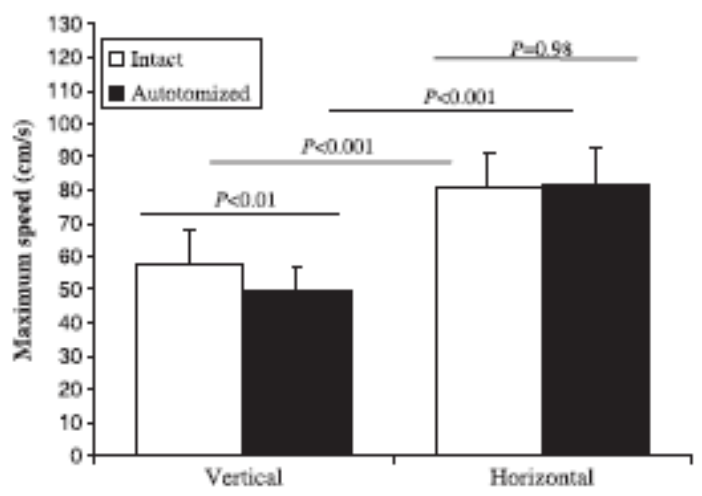

Fig. 2: Maximum speed $(\mathrm{cm} / \mathrm{s})$ for the vertical and the horizontal escape performance experiments as measured for intact Lygodactylus capensis and $2 \mathrm{~d}$ after tail autotomy 
(autotomized). p-Values originate from a Tukey-test following a repeated measures anova. Geckos were significantly faster on the horizontal platform than on the vertical platform. Intact geckos were significantly faster than autotomized geckos for the vertical experiment. Maximum speed did not differ between intact and autotomized geckos for the horizontal experiment. Values are mean \pm 1 SD.

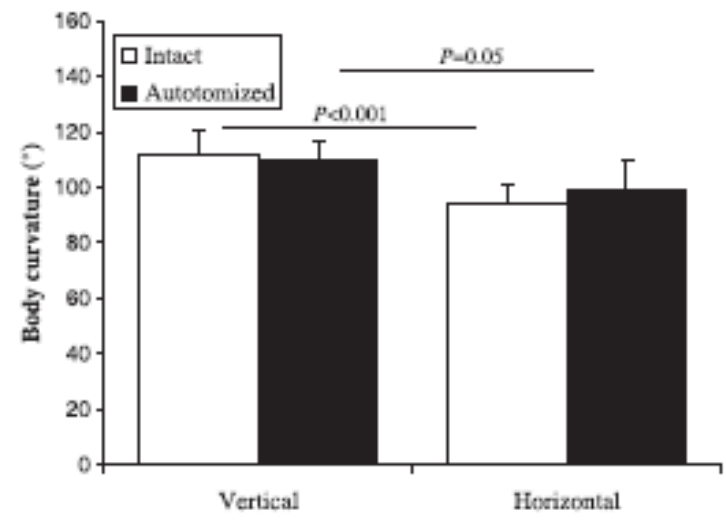

Fig. 3: Body curvature $\left(^{\circ}\right)$ for the vertical and the horizontal escape performance experiment as measured for intact Lygodactylus capensis and $2 \mathrm{~d}$ after tail autotomy (autotomized). p-Values originate from a Tukey-test following a repeated measures anova. Body curvature was significantly smaller on the horizontal platform than on the vertical platform for intact geckos but not for autotomized geckos. Body curvature did not differ between intact and autotomized geckos for both escape performance experiments and therefore, it is not indicated. Values are mean $\pm 1 \mathrm{SD}$.

The escape performance variables measured were not significantly different between the trials of each experiment after a SqBc (Table 6).

Table 6: Comparison of escape performance variables between the duplicate trials of the escape performance experiments (repeated measures anova)

\begin{tabular}{llll}
\hline Dependent variable & df & $F$ & p-Value \\
\hline Over all speed $(\mathrm{cm} / \mathrm{s})$ & 1,15 & 2.95 & 0.11 \\
Maximum speed $(\mathrm{cm} / \mathrm{s})$ & 1,37 & 1.80 & 0.19 \\
Inital speed $(\mathrm{cm} / \mathrm{s})$ & 1,33 & 0.82 & 0.37 \\
Body curvature $\left({ }^{2}\right)$ & 1,27 & 0.72 & 0.41 \\
Stride length front legs 17$)$ & 1,24 & 3.49 & 0.07 \\
Stride length hind legs 19 & 1,27 & 4.67 & $0.04^{*}$ \\
\hline
\end{tabular}

p-Values which were no longer significant after a sequential Bonferroni correction are indicated with an asterisk $(*)$.

Significantly more autotomized geckos fell backwards than intact geckos $\left[\chi_{(\mathrm{df}=1)}^{2}=5.24, \mathrm{p}=0.02 ; \mathrm{n}=19\right]$ for the vertical escape performance experiment. 


\section{Discussion}

Caudal autotomy confers a cost on the escape performance of Lygodactylus capensis on a vertical surface as autotomized geckos were slower than intact geckos for all three speed variables measured (overall, maximum and initial speed). Our results are similar to the findings of Brown et al. (1995) who demonstrated a negative effect of tail loss on the arboreal locomotory performance of the wall lizard Podarcis muralis. Podarcis muralis, however, is an unspecialized climber which uses its tail for positioning itself in vegetation by coiling it around twigs. In contrast, L. capensis is a specialized climber which is able to run even on smooth vertical surfaces because of adhesive foot pads containing thousands of setae which attach and detach rapidly (Autumn 2006). The dramatic decrease of clinging ability after toe-clipping a lizard with adhesive feet (Anolis carolinensis) demonstrates the importance of these setae (Bloch \& Irschick 2004). Moreover, an additional adhesive pad on the tail tip in L. capensis, which both the original and regenerated tail possesses, is used as a fifth point for attachment during jumps between twigs or branches (Vitt \& Ballinger 1982). The adhesive hairs on the feet and the tail may play an important role on the smooth surface we used during the experiments and the observed patterns may differ on other structures where geckos are able to use their claws (Vanhooydonck et al. 2005). During climbing, the feet of the gecko are attached to the surface which decreases the likelihood of lateral swing by the hindquarters and, therefore, reduces over-bending of the body (Arnold 1984). Accordingly, the tail may not be needed as a counterbalance during climbing and may explain why body curvature did not increase on the vertical surface after tail autotomy.

The observed backward falling of the autotomized geckos may be explained by the absence of the tail which the geckos use to prop the body up against the vertical surface. With tail loss, the gecko loses the ability to keep its upper body against the surface. Once the front feet disengage from the surface the gecko will fall backwards. Furthermore, the loss of the adhesive tail tip appears to contribute to the geckos' inability to run normally on a vertical surface (Vitt \& Ballinger 1982). This may explain why speed on the vertical surface is not influenced by body curvature or stride length.

Lygodactylus capensis were much faster on the horizontal surface than the vertical surface: maximum speed was $40 \%$ higher for the intact geckos and $64 \%$ higher for the autotomized geckos on the horizontal surface than on the vertical surface. This increase in speed can be explained by the greater influence of gravity on a vertical surface. Interestingly, the geckos did not increase their stride length to increase their speed on either the vertical or the horizontal surface. This is in contrast to the observations of Martín \& Avery (1998) who found that Psammodromus algirus increased speed by increasing stride length. The greater body curvature of the intact geckos on a horizontal surface may therefore contribute to the higher speed. Furthermore, initial speed of the autotomized geckos was much faster on the horizontal than the vertical surface but this difference was not evident for intact geckos. Although initial speed may be a weak measure of escape performance, this may show a further impact of autotomy on the first important seconds of escape on a vertical surface. 
The geckos demonstrated jumping (when no feet were touching the surface) when running on the horizontal surface, but this was not observed on the vertical platform. This corresponds with the restraints of climbing: geckos should not be able to jump on a vertical surface as it is essential to have at least one foot in contact with the surface during climbing (see above; Zaaf et al. 2001). However, many ground-dwelling lizards show jumping, especially at higher speeds (Van Damme et al. 1998; Zaaf et al. 2001). Nevertheless, Zaaf et al. (2001) did not demonstrate jumping during the horizontal locomotion of the tokay gecko Gekko gecko, a specialized climber with adhesive foot pads similar to those of L. capensis. The smaller body size of L. capensis ( $1.1 \mathrm{~g}$; this study) may allow jumping during horizontal locomotion in contrast to the more heavy-bodied G. gecko (53.2 g; Zaaf et al. 2001).

Escape speed may increase on a horizontal surface after autotomy if the tail does not play an obvious role during locomotion or if the tail hinders locomotion because of the friction with the substrate and lag from additional mass (Daniels 1983). However, the tail plays an important role in horizontal locomotion in many lizards and is often used as a counterbalance to the body to get weight concentrated over the hind limbs which are a main source of forward propulsion during running (Arnold 1984, 1988). A decrease in escape speed with tail loss was found for P. algirus (Martín \& Avery 1998) and the ground skink, Scincella lateralis (Formanowicz et al. 1990). However, we found no difference in horizontal escape speed of L. capensis between intact and autotomized geckos indicating that the tail may not play an apparent role during horizontal locomotion. Nevertheless, the geckos showed differences in stride length between the front and hind legs on the horizontal surface. The stride length of the hind legs was not influenced by autotomy while the stride length of the front legs was shorter in autotomized geckos than in intact geckos, although this difference was not significant. This may indicate that the hind legs are used for forward propulsion and the tail is used to distribute weight to the hind legs and to relieve the front legs which are then able to make greater strides. The speed should therefore decrease with tail loss, but this is perhaps compensated for by the loss of tail mass or loss of friction with the surface as the tail comprises half of the total body length and $13 \%$ of the total body mass for L. capensis (this study). However, stride length was very inconsistent within geckos between repeats on the horizontal surface, probably because of the varying locomotory patterns including jumping used on the horizontal surface. Further investigations, such as lateral filming of the running, are therefore needed to examine the horizontal locomotion of this gecko in detail.

On the horizontal surface, body curvature did not differ between intact and autotomized geckos and therefore, the tail may not be used to counterbalance the lateral movement of the body, as L. capensis are light-bodied. Farley \& Ko (1997) demonstrated that the lateral body movement has only a small energetic influence during locomotion, and therefore, the use of the tail as counterbalance may not be very important. Nevertheless, body curvature of the intact geckos was found to be greater on a horizontal than a vertical surface but body curvature did not influence speed. The small amount of variation in body curvature within an experiment may yet be the reason why no relationship between speed and body curvature was found for the two escape performance experiments. Additional studies with a larger sample size are required to explicitly determine the contribution of body curvature to a change in speed for these geckos. 
Brown et al. (1995) determined that P. muralis with regenerated tails were faster and showed a superior performance after autotomy than individuals which possessed an original tail before autotomy. They suggested that individuals with regenerated tails had already gained experience in running without a tail and were therefore better able to run when their tails were autotomized a second time. This hypothesis must be rejected for L. capensis as a difference in locomotion after autotomy was not found between individuals that possessed original tails and individuals that possessed regenerated tails before experimental autotomy.

We found that the original tails were significantly longer than regenerated tails but no influence of tail length on locomotion was established. However, a specific tail length may be needed for flawless locomotion on a vertical surface. The geckos are able to regenerate the tail rapidly (onset of regeneration: $11 \mathrm{~d}$ post-autotomy; K. Medger, pers. obs.) and it was found that the adhesive tail tip in Lygodactylus was regenerated after 4 wk (Vitt \& Ballinger 1982). In our observed population of L. capensis, 57\% were found to have regenerated their tails which suggests a high predation risk for this population. It is therefore likely that both the high autotomy frequency and the fast tail regeneration are an adaptation to the high predation pressure faced by this species (see also Vitt \& Ballinger 1982). Furthermore, the high rate of tail regeneration which these geckos experience may keep the costs resulting from autotomy relatively low. The difficulties that the autotomized geckos demonstrated when running on a vertical surface, which were not evident on a horizontal surface, may ultimately influence the behaviour of the geckos in a natural situation. It is possible that autotomized L. capensis selectively utilize denser and more horizontal habitats to avoid predation by hiding and allow for faster fleeing during escape. Smooth surfaces, similar to that used in this study, can be found in the suburban habitat utilized by these geckos, for example, lamp poles or drainpipes. Naturally occurring smooth surfaces utilized by these geckos include certain rock formations, broad leaves or smooth bark. The effects of autotomy on locomotion may, however, not be as great in a completely natural setting where the geckos are able to use their claws on rough surfaces. Nevertheless, a change of habitat and cryptic anti-predator behaviour because of the locomotory influence of autotomy may prove costly through a decrease in territory size, less time spent foraging and reduced access to mates. Autotomized individuals of the keeled earless lizard, Holbrookia propinqua, for example, stay closer to plant cover than individuals with intact tails (Cooper 2003). Autotomized Iberian rock lizards, Lacerta monticola, use more rocky habitats and move as little as possible to avoid detection by predators (Martín \& Salvador 1992). The change of microhabitat use by L. monticola can also result in decreased food availability and diet diversity (Martín \& Salvador 1993). Caudal autotomy of male Psammodromus algirus resulted in a reduction of home range size and might therefore affect the access to females (Salvador et al. 1995). Further studies should examine habitat and behavioural changes in autotomized geckos such as L. capensis and their susceptibility to predation. 


\section{Literature Cited}

- Arnold, E. N. 1984: Evolutionary aspects of tail shedding in lizards and their relatives. J. Nat. Hist. 18, 127-169.

- Arnold, E. N. 1988: Caudal autotomy as a defence. In: Biology of the Reptilia, Vol. 13 (Gans, C. \& Huey, R., eds). Alan R. Liss, New York, pp. 235-273.

- Autumn, K. 2006: How gecko toes stick. Am. Sci. 94, 124-132.

- Autumn, K., Liang, Y. A., Hsieh, S. T., Zesch, W., Chan, W. P., Kenny, T. W., Fearing, R. \& Full, R. J. 2000: Adhesive force of a single gecko foot-hair. Nature 405, 681-685.

- Bateman, P. W. \& Fleming, P. A. 2005: Direct and indirect costs of limb autotomy in field crickets Gryllus bimaculatus. Anim. Behav. 69, 151-159.

- Bauer, A. M. 1998: Morphology of the adhesive tail tips of carphodactyline geckos (Reptilia: Diplodactylidae). J. Morphol. 235, 41-58.

- Bloch, N. \& Irschick, D. J. 2004: Toe-clipping dramatically reduces clinging performance in a pad-bearing lizard (Anolis carolinensis). J. Herpetol. 37, 293-298.

- Brown, R. M., Taylor, D. H. \& Gist, D. H. 1995: Effect of caudal autotomy on locomotor performance of wall lizards (Podarcis muralis). J. Herpetol. 29, 98-105.

- $\quad$ Brueseke, M. A., Rypstra, A. L., Walker, S. E. \& Persons, M. H. 2001: Leg autotomy in the wolf spider Pardosa milvina: a common phenomenon with few apparent costs. Am. Midl. Nat. 146, 153-160.

- Chapple, D. G., McCoull, C. J. \& Swain, R. 2004: Effect of tail loss on sprint speed and growth in newborn skinks, Niveoscincus metallicus. J. Herpetol. 38, $137-140$.

- Chapple, D. G. \& Swain, R. 2002: Effect of caudal autotomy on locomotor performance in a viviparous skink, Niveoscincus metallicus. Funct. Ecol. 16, $817-825$.

- Congdon, J. D., Vitt, L. J. \& King, W. W. 1974: Geckos: adaptive significance and energetics of tail autotomy. Science 184, 379-1380.

- Cooper, W. E. 2003: Shifted balance of risk and cost after autotomy affects use of cover, escape, activity, and foraging in the keeled earless lizard (Holbrookia propinqua). Behav. Ecol. Sociobiol. 54, 179-187.

- Daniels, C. B. 1983: Running: an escape strategy enhanced by autotomy. Herpetologica 39, 162-165.

- Daniels, C. B. 1985: The effect of tail autotomy on the exercise capacity of the water skink, Sphenomorphus quoyii. Copeia 4, 1074-1077.

- Downes, S. \& Shine, R. 2001: Why does tail loss increase a lizard's later vulnerability to snake predators? Ecology 82, 1293-1303.

- Dubost, G. \& Gasc, J.-P. 1987: The process of total tail autotomy in the South American rodent, Proechimys. J. Zool. (Lond.) 212, 563-572.

- Ducey, P. K., Brodie, E. D. Jr \& Baness, E. A. 1993: Salamander tail autotomy and snake predation: role of antipredator behaviour for three neotropical Bolitoglossa (Caudata: Plethodontidae). Biotropica 25, 344-349.

- Farley, C. T. \& Ko, T. C. 1997: Mechanics of locomotion in lizards. J. Exp. Biol. 200, 2177-2188.

- Formanowicz, D. R. Jr 1990: The antipredator efficacy of spider leg autotomy. Anim. Behav. 40, 400—409. 
- Formanowicz, D. R. Jr, Brodie, E. D. Jr \& Bradley, P. J. 1990: Behavioural compensation for tail loss in the ground skink, Scincella lateralis. Anim. Behav. 40, 782-784.

- Fox, S. F. \& McCoy, J. K. 2000: The effects of tail loss on survival, growth, reproduction, and sex ration of offspring in the lizard Uta stansburiana in the field. Oecologia 122, 327-334.

- Fox, S. F. \& Rostker, M. A. 1982: Social cost of tail loss in Uta stansburiana. Science 218, 692-693.

- Juanes, F. \& Smith, L. D. 1995: The ecological consequences of limb damage and loss in decapod crustaceans: a review and prospectus. J. Exp. Mar. Biol. Ecol. 193, 197-223. Links

- Langkilde, T. \& Shine, R. 2006: How much stress do researchers inflict on their study animals? A case study using a scincid lizard, Eulamprus heatwolei J. Exp. Biol. 209, 1035-1043.

- Maginnis, T. L. 2006: The costs of autotomy and regeneration in animals: a review and framework for future research. Behav. Ecol. 17, 857-872.

- Maiorana, V. C. 1977: Tail autotomy, functional conflicts and their resolution by a salamander. Nature $265,533-535$.

- Martín, J. \& Avery, R. A. 1998: Effects of tail loss on the movement patterns of the lizard, Psammodromus algirus. Funct. Ecol. 12, 794-802. Links

- Martín, J. \& Salvador, A. 1992: Tail loss consequences on habitat use by the Iberian rock lizard, Lacerta monticola. Oikos 65, 328-333.

- Martín, J. \& Salvador, A. 1993: Tail loss reduces mating success in the Iberian rock-lizard, Lacerta monticola. Behav. Ecol. Sociobiol. 32, 185-189.

- McConnachie, S. \& Whiting, M. J. 2003: Costs associated with tail autotomy in an ambush foraging lizard, Cordylus melanotus melanotus. Afr. Zool. 38, $57-65$.

- McKee, R. C. \& Adler, G. H. 2002: Tail autotomy in the Central American spiny rat, Proechimys semispinosus. Stud. Neotrop. Fauna Environ. 37, 181185.

- Pianka, E. R. \& Huey, R. B. 1978: Comparative ecology, resource utilization and niche segregation among gekkonid lizards in the southern Kalahari. Copeia 1978, 691-701.

- Ramsay, K., Kaiser, M. J. \& Richardson, C. A. 2001: Invest in arms: behavioural and energetic implications of multiple autotomy in starfish (Asterias rubens). Behav. Ecol. Sociobiol. 50, 360-365.

- Rice, W. R. 1989: Analyzing tables of statistical tests. Evolution 43, 223 225.

- Salvador, A., Martín, J. \& López, P. 1995: Tail loss reduces home range size and access to females in male lizards, Psammodromus algirus. Behav. Ecol. 6, $382-387$.

- Statsoft, 2001: Statsoft Inc., 6th ed., Tulsa, U.S.A.

- Stoks, R. 1998: Effect of lamellae autotomy on survival and foraging success of the damselfly Lestes sponsa (Odonata: Lestidae). Oecologia 117, 443-448.

- Van Damme, R., Aerts, P. \& Vanhooydonck, B. 1998: Variation in morphology, gait characteristics and speed of locomotion in two populations of lizards. Biol. J. Linn. Soc. 63, 409-427.

- Vanhooydonck, B., Andronescu, A., Herrel, A. \& Irschick, D. J. 2005: Effects of substrate structure on speed and acceleration capacity in climbing geckos. Biol. J. Linn. Soc. 85, 385-393. 
- Vitt, L. J. \& Ballinger, R. E. 1982: The adaptive significance of a complex caudal adaptation in the tropical gekkonid lizard, Lygodactylus klugei. Can. J. Zool. 60, 2582-2587.

- Vitt, L. J., Congdon, J. D. \& Dickson, N. A. 1977: Adaptive strategies and energetics of tail autotomy in lizards. Ecology 58, 326-337.

- Vitt, L. J. \& Cooper, W. E. 1986: Tail loss, tail color, and predator escape in Eumeces (Lacertilia: Scincidae): age-specific differences in costs and benefits. Can. J. Zool. 64, 583-592.

- Wasson, K., Lyon, B. E. \& Knope, M. 2002: Hair-trigger autotomy in porcelain crabs is a highly effective escape strategy. Behav. Ecol. 13, 481486.

- Zaaf, A., van Damme, R., Herrel, A. \& Aerts, P. 2001: Spatio-temporal gait characteristics of level and vertical locomotion in a ground-dwelling and a climbing gecko. J. Exp. Biol. 204, 1233-1246. 